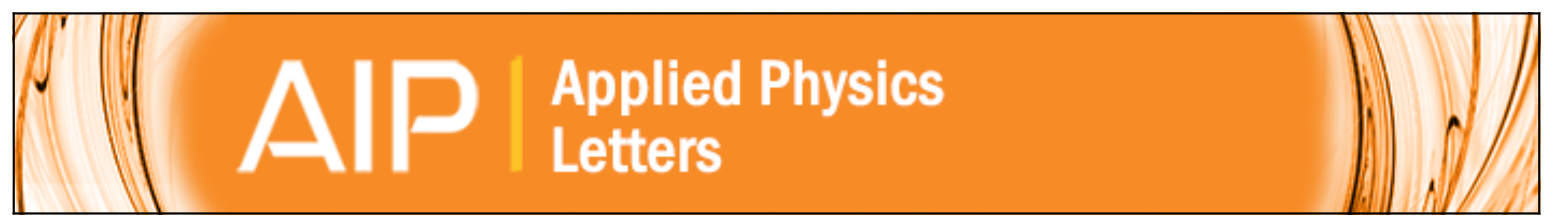

\title{
Enhanced magnetic response of fluids using self-assembled petal-like iron oxide particles
}

You-Hwan Son, Jung-Kun Lee, Yee Soong, Donald Martello, and Minking Chyu

Citation: Applied Physics Letters 96, 121905 (2010); doi: 10.1063/1.3371713

View online: http://dx.doi.org/10.1063/1.3371713

View Table of Contents: http://scitation.aip.org/content/aip/journal/apl/96/12?ver=pdfcov

Published by the AIP Publishing

\section{Articles you may be interested in}

Loading and release of internally self-assembled emulsions embedded in a magnetic hydrogel Appl. Phys. Lett. 104, 043701 (2014); 10.1063/1.4862811

Biological colloid engineering: Self-assembly of dipolar ferromagnetic chains in a functionalized biogenic ferrofluid

Appl. Phys. Lett. 101, 063701 (2012); 10.1063/1.4742329

Magnetically tunable self-assembly of colloidal rings

Appl. Phys. Lett. 97, 083105 (2010); 10.1063/1.3483137

Investigation on configuration of self-assembly in magnetic fluid film under a magnetic field J. Appl. Phys. 106, 014501 (2009); 10.1063/1.3157209

Self-assembly and magnetism in core-shell microspheres

J. Vac. Sci. Technol. A 21, 1515 (2003); 10.1116/1.1564031

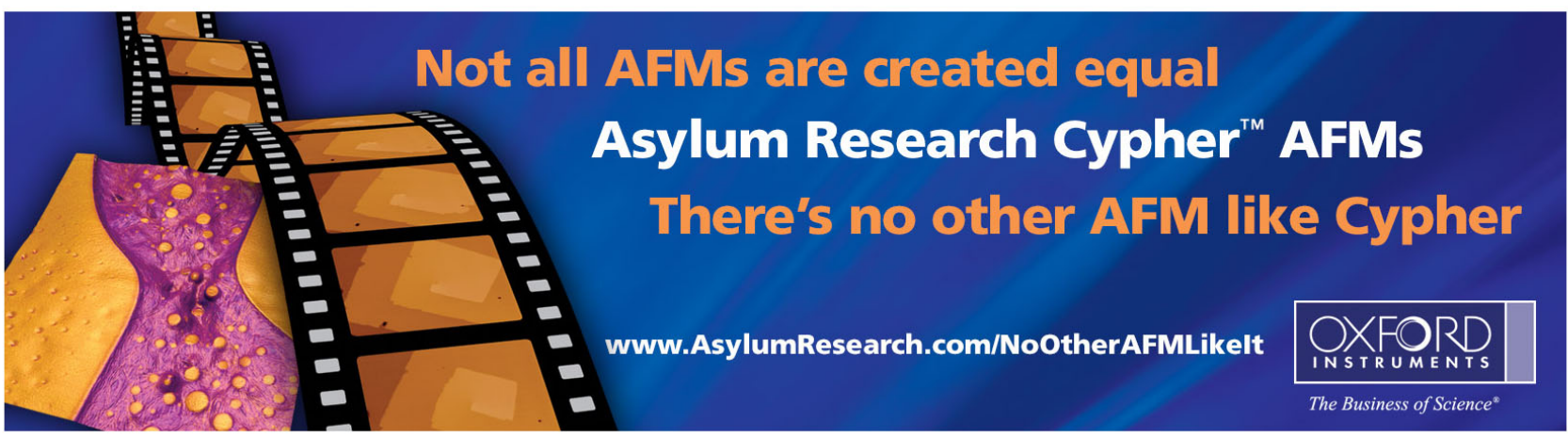




\title{
Enhanced magnetic response of fluids using self-assembled petal-like iron oxide particles
}

\author{
You-Hwan Son, ${ }^{1}$ Jung-Kun Lee, ${ }^{1, a)}$ Yee Soong, ${ }^{2}$ Donald Martello, ${ }^{2}$ and Minking Chyu ${ }^{1}$ \\ ${ }^{1}$ Department of Mechanical Engineering \& Material Science, University of Pittsburgh, Pittsburgh, \\ Pennsylvania 15261, USA \\ ${ }^{2}$ National Energy Technology Laboratory, Pittsburgh, Pennsylvania 15236, USA
}

(Received 8 January 2010; accepted 25 February 2010; published online 23 March 2010)

\begin{abstract}
Using self-assembled iron oxide (SAIO) particles with petal-like morphology, aqueous fluids containing magnetic particles were prepared and the effect of hierarchical particle surface on the viscoelasticity under magnetic was investigated. The fluids consisting of self-assembled iron oxide particles exhibit highly tunable viscoelasticity which is controlled by applying external magnetic field. A difference between SAIO particles and spherical particles is explained by the fact that surface features of the self-assembled particles increased the network strength between particles in the fluids. () 2010 American Institute of Physics. [doi:10.1063/1.3371713]
\end{abstract}

Magnetic materials have attracted considerable attentions because of their tunable properties in biological and engineering applications. ${ }^{1,2}$ One of their interesting properties is that the magnetic particles in solutions can be aligned when an external magnetic field is applied. Such solutions containing magnetic materials, which are so called magnetorheological fluids or ferrofluids depending on the size of magnetic particles, are regarded as one of the smart materials. Their viscoelastic properties can be rapidly and reversibly controlled by the magnetic field. Therefore, the solutions show dramatic and tunable changes from a fluid-like to a solid-like state within milliseconds under the influence of magnetic field. ${ }^{3}$ This tunable rheology is intensively investigated for their use in biomedical and mechanical application such as targeted drug delivery, biomolecule separation, and heat transfer. ${ }^{2,4}$

Recently, the separation methods using magnetic particle have been developed to easily isolate target biomolecules from sample solution with minimum efforts. This direct separation of biomolecules may save resources and time, compared with traditional separation methods. ${ }^{3,5}$ Once target biological cells or molecules are immobilized on the magnetic nanoparticles, they can be easily separated from a sample solution and efficiently transferred to a desired location by controlling the intensity and distribution of the magnetic field. Also, the large surface area of the magnetic particles gives a large number of binding sites, resulting in large capacity of biomolecule separation.

In the magnetic fluids, important phenomena are interparticle interaction and material adsorption that occur on the surface of the magnetic particles. Therefore, the surface morphology of the magnetic particles is one of the most important aspects that determine the functionalities of magnetic particles in the fluids. To date, however, the spherical magnetic nanoparticle such as carbonyl iron, magnetite $\left(\mathrm{Fe}_{3} \mathrm{O}_{4}\right)$, maghemite $\left(\gamma-\mathrm{Fe}_{2} \mathrm{O}_{3}\right)$ particles or beads containing magnetic multicores with different surface layer have been mainly used as a stimuli-responsive material under magnetic field. ${ }^{6-8}$

\footnotetext{
a) Author to whom correspondence should be addressed. Electronic mail: jul37@pitt.edu.
}

In this study, we have examined the magnetorheological behavior of nonspherical particles that have hierarchical structure and large surface area with an emphasis on the effect of the surface morphology on the viscoelastic properties of fluids under magnetic field. Magnetic particles with a petal-like morphology were synthesized by using the selfassembly of nanoparticles via an ethylene glycol (EG) mediated reaction. This EG mediated method has been widely used to produce particles with controlled morphologies such $\mathrm{V}_{2} \mathrm{O}_{5}$ hollow microspheres, $\mathrm{SnO}$ nanowires, cobalt alkoxide layered particles and flowerlike iron oxide particles. ${ }^{9-12}$ Selfassembled iron oxide particles with nanometer size surface features were synthesized using high purity $\mathrm{Fe}(\mathrm{NO})_{3} \cdot 6 \mathrm{H}_{2} \mathrm{O}$, urea, and ethylene glycol.

Self-assembled iron oxide particles with petal-like morphology were dispersed in water. SAIO particles were well dispersed in water using the surfactants. Aqueous fluids containing $30 \mathrm{wt} \%$ of SAIO particles did not show flocculation over a week. High stability of SAIO fluids is attributed to the open pore structure of SAIO particles, which helps their Brownian motion and prevents agglomeration. The rheological properties of aqueous fluids were characterized using a rheometer (MCR-301, Anton Paar) with magneto-rheological devices.

A scanning electron microscope (SEM) image of asgrown SAIO particles is shown in Fig. 1(a). Thin plates were randomly assembled to make flowerlike iron-oxide particles with hierarchical surfaces. The size of SAIO particles is around $1 \mu \mathrm{m}$ in diameter. Particles have very rough surfaces consisting of $40-60 \mathrm{~nm}$ thick distorted plates. This unique morphology with large surface area results from the selfassembly of iron hydroxide nanoparticle through the coordination of $\mathrm{Fe}\left(\mathrm{NO}_{3}\right)$ with EG. Transmission electron microscope (TEM) images of SAIO particles before and after thermal annealing are shown in Figs. 1(c) and 1(d). The individual thin petal plate of as-grown SAIO particles is composed of interconnected nanoparticles with a diameter of $15 \mathrm{~nm}$. During the thermal annealing, the nanoparticles did not grow significantly and the shape of SAIO particles was maintained. XRD patterns for before and after annealed particles were shown in Fig. 1(d). As-grown SAIO particles contain residual organic components and their crystal struc- 

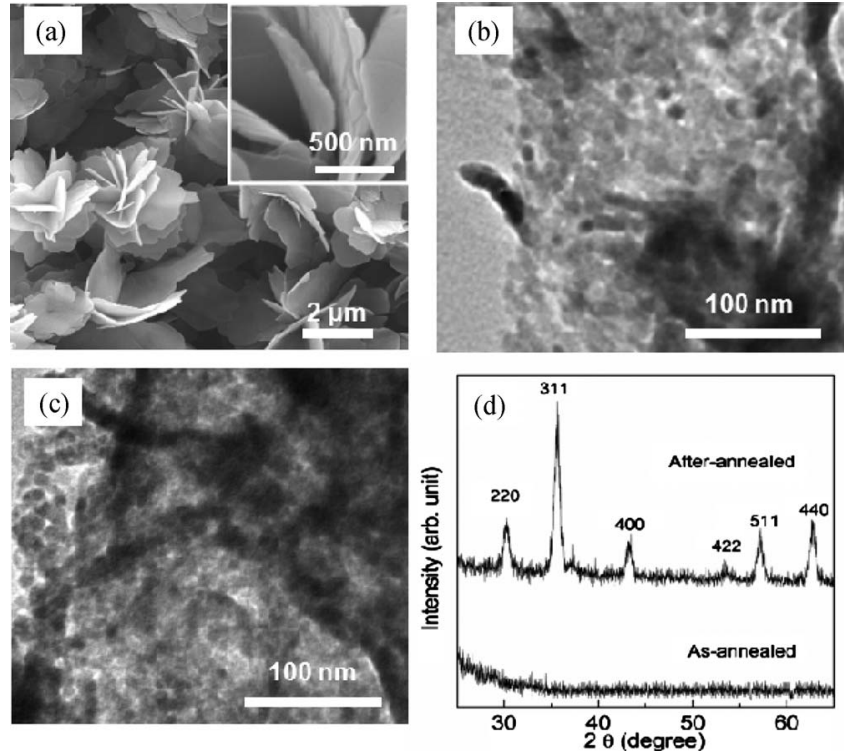

FIG. 1. (a) SEM image of as-grown self-assembled iron oxide particle via EG mediated synthetic route, (b) TEM image of as-grown self-assembled iron oxide particle, (c) TEM image of self-assembled iron oxide particle after thermal annealing, (d) XRD pattern of self-assembled iron oxide particle after annealing at $400{ }^{\circ} \mathrm{C}$ with $\mathrm{N}_{2}$.

ture was amorphous. After thermal annealing, the color of the nanoparticles turned to be black and the diffraction peaks of annealed nanoparticles agree well with those of magnetite $\left(\mathrm{Fe}_{3} \mathrm{O}_{4}\right.$, JCPDS 85-1436). This result indicates that individual nanoparticles are crystallized from amorphous to magnetite without any morphological change during annealing. M-H curve of thermally annealed SAIO particles was measured at room temperature using a vibrating sample magnetometer. As shown Fig. 2(a), a clear magnetic hysteresis loop was observed. The saturated magnetization $\left(\sigma_{\mathrm{s}}\right)$ of SAIO particles is $72 \mathrm{emu} / \mathrm{g}$, which is very close to that of bulk maghemite $\left(\gamma-\mathrm{Fe}_{2} \mathrm{O}_{3}\right)(80 \mathrm{emu} / \mathrm{g})$ or magnetite $\left(\mathrm{Fe}_{3} \mathrm{O}_{4}\right)$ $(92 \mathrm{emu} / \mathrm{g}) .{ }^{13,14}$ A very small coercive field in Fig. 2 supports that the microsized SAIO particles consist of the nanosized magnetic particles with the diameter of $15 \mathrm{~nm}$. At this size, the thermal fluctuation is large enough to overcome the magnetic anisotropy of the nanoparticles under zero magnetic field and significantly reduce the coercive field.

To understand an influence of hierarchical morphology of SAIO particles on the rheology of the fluids containing magnetic particles, we carried out magnetorheological analysis in a strain amplitude sweep mode. Figure 2(b) shows the
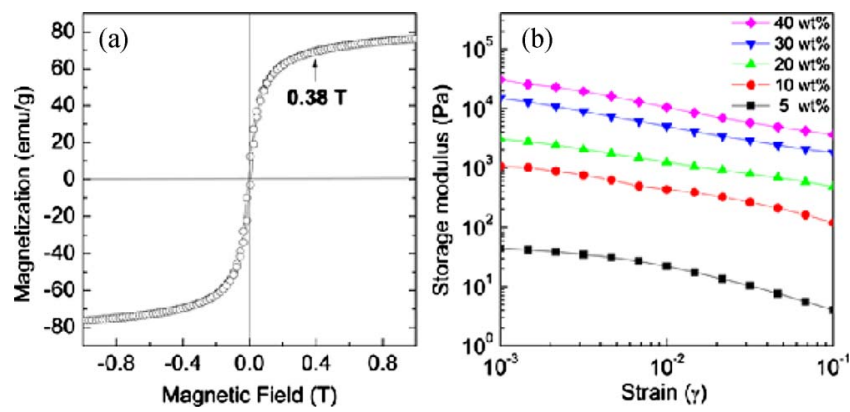

FIG. 2. (Color online) (a) M-H curves of thermally SAIO particles, (b) Storage modulus vs strain amplitude curves of the fluid fluids containing different amount of SAIO particles when the magnetic field of $0.38 \mathrm{~T}$ is applied.
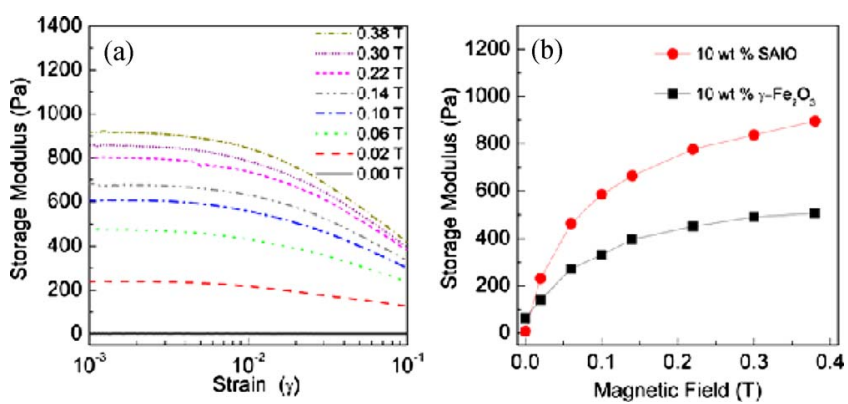

FIG. 3. (Color online) (a) Storage modulus vs strain amplitude curves of the fluids containing 10 wt $\%$ SAIO particles under different magnetic field $(0-0.38 \mathrm{~T})$, (b) A change in the storage modulus of the fluids containing 10 wt $\%$ SAIO particles or spherical $\gamma$ - $\mathrm{Fe}_{2} \mathrm{O}_{3}$ particles.

plot of the storage modulus versus the strain amplitude in the fluids containing a different amount of SAIO particles. The storage modulus increases dramatically as the content of SAIO particles increased from 5 to $40 \mathrm{wt} \%$.

Figure 3(a) shows an increment of the storage modulus in a $10 \mathrm{wt} \%$ fluid as a function of magnetic field. When the magnetic field of $0.38 \mathrm{~T}$ was applied, the storage modulus of the fluid containing the SAIO particles increased by more than two orders of the magnitude, compared to zero magnetic field. As a control sample, we also prepared a fluid containing spherical $\gamma-\mathrm{Fe}_{2} \mathrm{O}_{3}$ particles with an average diameter of $30 \mathrm{~nm}$. Their saturated magnetization was about $80 \mathrm{emu} / \mathrm{g}$, which is very similar to that of SAIO particles. The stability of the fluid containing 10 wt $\% \gamma-\mathrm{Fe}_{2} \mathrm{O}_{3}$ nanoparticles is similar to that of the SAIO particle fluid due to the nanosize of $\gamma-\mathrm{Fe}_{2} \mathrm{O}_{3}$ particles. Same analysis was performed using the fluids of spherical $\gamma-\mathrm{Fe}_{2} \mathrm{O}_{3}$ particles to examine the effect of the surface morphology. Though the saturated magnetizations of $\gamma-\mathrm{Fe}_{2} \mathrm{O}_{3}$ and SAIO particles are almost same, their storage moduli are very different. When 10 wt $\%$ of the spherical particles were added, there is a moderate increase in the storage modulus under the magnetic field, which is previous studies. ${ }^{4,15,16}$ At same solid content and magnetic field, as shown in Fig. 3(b), the storage modulus of SAIO fluids is two times as large as that of the spherical particles. A comparison of two fluids indicates that a unique morphology of SAIO particles increases interparticle interaction at the surface, leading to larger friction between the particles and enhanced dependency on the magnetic field.

The strength of the interparticle interaction and the particle networks in the fluids can be evaluated by monitoring the transition of the fluids from a solid-like status to a liquid-like status. Therefore, we forced two different fluids (10 wt $\%$ SAIO particle fluid and $10 \mathrm{wt} \%$ spherical $\gamma-\mathrm{Fe}_{2} \mathrm{O}_{3}$ particle fluid) to be subject to incremental oscillatory motion and compared their loss modulus and storage modulus as a function of oscillatory strain. Figure 4(a) shows the change in the storage $\left(G^{\prime}\right)$ and loss modulus $\left(G^{\prime \prime}\right)$ obtained from the oscillatory measurement at a constant magnetic field of $0.38 \mathrm{~T}$. As the amplitude of the oscillatory strain increases, the network of the magnetic particles is shaken and the strength of interparticle connection is weakened. When the oscillatory strain reaches a critical strain, the breakdown of the particle network begins and $G^{\prime}$ starts to fall significantly. At the same critical strain, $G^{\prime \prime}$ exhibits a maximum value due to the out-of-phase motion of the particle network in the fluids. These changes in $G$ and $G^{\prime \prime}$ at a critical point represent 

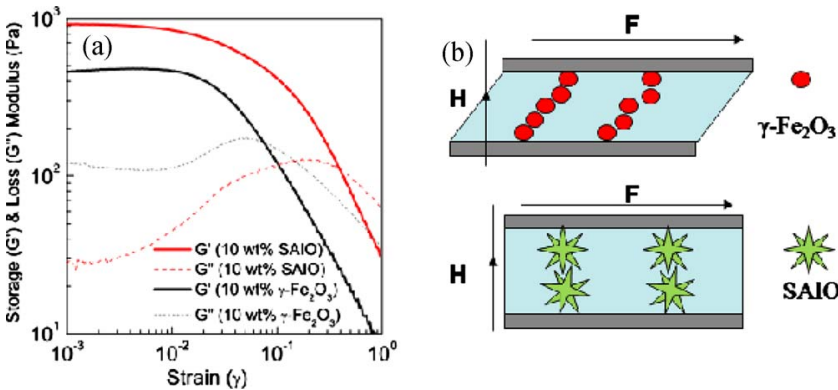

SAIO

FIG. 4. (Color online) (a) Storage $\left(\mathrm{G}^{\prime}\right)$ and Loss $\left(\mathrm{G}^{\prime \prime}\right)$ modulus for SAIO ferrofluid and spherical $\gamma-\mathrm{Fe}_{2} \mathrm{O}_{3}$ at $0.38 \mathrm{~T}$, (b) Scheme for increasing stress to break the rows of aligned particles by the rugged surface of SAIO.

yield of fluids over applied strain. As the oscillatory strain becomes larger than critical strain $\left(\gamma_{y}\right)$, the fluids show a transition from solid-like state to liquid-like state. ${ }^{17,18}$ The strength of the magnetic particle network is estimated by comparing the critical strain $\left(\gamma_{y}\right)$ and effective yield stress $\left(\tau_{y}\right)$ of colloidal fluids. $G^{\prime}$ corresponding to the critical strain is $G_{y}^{\prime}$. By using $\gamma_{y}$ and $G_{y}^{\prime}$, we estimate the effective yield stress which induces a viscous flow that is indicative of a liquidlike state,

$$
\begin{aligned}
& \tau_{y}=\gamma_{y} G_{y}^{\prime}, \\
& \tau_{i}=\gamma_{i} G^{\prime}(\gamma \rightarrow 0) .
\end{aligned}
$$

In additions, $G^{\prime}$ in low strain region, $G^{\prime}(\gamma \rightarrow 0)$ and initial strain $\left(\gamma_{i}\right)$ show stress $\left(\tau_{i}\right)$ that is required for the initial breakdown of the colloidal network. The initial strain is defined as a strain of a point where linear viscoelastic region ends. Then, $\tau_{i}$ represents the strength of the particle network in the solid-like state. Table I shows values of rheological parameters to estimate the strength of colloidal network at $0.38 \mathrm{~T}$. The value $\tau_{y}$, stress of self-assembled particle is about five times stronger than that of spherical $\mathrm{Fe}_{2} \mathrm{O}_{3}$ particles, attesting that rugged surface of the self-assembled particles increases interparticle friction when they are aligned

TABLE I. Rheological parameters of SAIO and spherical $\gamma-\mathrm{Fe}_{2} \mathrm{O}_{3}$ particle fluids at a constant magnetic field of $0.38 \mathrm{~T}$.

\begin{tabular}{cccrccc}
\hline \hline Fluid & $\mathrm{G}_{y}^{\prime} / \mathrm{Pa}$ & $\gamma_{y}$ & $\tau_{y} / \mathrm{Pa}$ & $\mathrm{G}_{(y \rightarrow 0)}^{\prime} / \mathrm{Pa}$ & $\gamma_{i}$ & $\tau_{i} / \mathrm{Pa}$ \\
\hline $10 \mathrm{wt} \% \mathrm{SAIO}$ & 254 & 0.21 & 53.34 & 906 & 0.005 & 4.53 \\
$10 \mathrm{wt} \% \mathrm{Fe}_{2} \mathrm{O}_{3}$ & 186 & 0.052 & 9.67 & 473 & 0.006 & 2.84 \\
\hline \hline
\end{tabular}

and neighbored by the external magnetic field. This is schematically explained in Fig. 4(b). The values of $\tau_{i}$, show that the self-assembled particles and the spherical particles begin to break the network structure over 4.53 and $2.84 \mathrm{~Pa}$. These results are consistent with the previous explanation that SAIO particles are more rigid to strain amplitude. Therefore, it is more difficult to make a transition to the liquidlike state that exhibit viscous flow under oscillatory motion, leading to larger effective yield stress of the SAIO particle fluid.

In summary, self-assembled petal-like iron oxide particles with rough surface provide enhanced controllability and increased colloidal network strength under external magnetic field. SAIO fluids demonstrate higher modulus strength and effective yield stress than spherical particle fluids. This suggests that hierarchical surfaces of SAIO particles interlock under magnetic field and form a strong network structure.

This work was supported by the U.S. Department of Energy through National Energy Technology Laboratory (NETL).

${ }^{1}$ E. M. Furst and A. P. Gast, Phys. Rev. E 61, 6732 (2000).

${ }^{2}$ A. K. Gupta and M. Gupta, Biomaterials 26, 3995 (2005).

${ }^{3}$ J. M. Ginder and L. C. Davis, Appl. Phys. Lett. 65, 3410 (1994).

${ }^{4}$ P. D. Shima, J. Philip, and B. Raj, Appl. Phys. Lett. 95, 133112 (2009).

${ }^{5}$ J.-W. Choi, in Fabrication of Micromachined Magnetic Particle Separators for Bioseparation in Microfluidic Systems: Methods in Molecular Biology, edited by S. D. Minteer (Springer, New York, 2005).

${ }^{6}$ B. J. Park, K. H. Song, and H. J. Choi, Mater. Lett. 63, 1350 (2009)

${ }^{7}$ Y. Lu, Y. Yin, B. T. Mayers, and Y. Xia, Nano Lett. 2, 183 (2002).

${ }^{8}$ Y.-X. Wang, S. Hussain, and G. Krestin, Eur. Radiol. 11, 2319 (2001).

${ }^{9}$ A. M. Cao, J. S. Hu, H. P. Liang, and L.-J. Wan, Angew. Chem., Int. Ed. 44, 4391 (2005).

${ }^{10}$ L. S. Zhong, J. S. Hu, A. M. Ca, Q. Liu, W.-G. Song, and L.-J. Wan, Chem. Mater. 19, 1648 (2007).

${ }^{11}$ A. M. Cao, J. S. Hu, H. P. Liang, W.-G. Song, L.-J. Wan, X.-L. He, X.-G. Gao, and S.-H. Xia, J. Phys. Chem. B 110, 15858 (2006).

${ }^{12}$ L. S. Zhong, J. S. Hu, H. P. Liang, A.-M. Cao, W.-G. Song, and L.-J. Wan, Adv. Mater. 18, 2426 (2006)

${ }^{13}$ S. Sun and H. Zeng, J. Am. Chem. Soc. 124, 8204 (2002).

${ }^{14}$ J. Park, E. Lee, N.-M. Hwang, M. Kang, S. C. Kim, Y. Hwang, J.-G. Park, H.-J. Noh, J.-Y. Kim, J.-H. Park, and T. Hyeon, Angew. Chem., Int. Ed. 44, 2872 (2005).

${ }^{15}$ J. de Vicente, J. P. Segovia-Gutiérrez, E. Andablo-Reyes, F. Vereda, and R. Hidalgo-Álvarez, J. Chem. Phys. 131, 194902 (2009).

${ }^{16}$ R. C. Bell, J. O. Karli, A. N. Vavreck, D. T. Zimmerman, G. T. Ngatu, and N. M. Wereley, Smart Mater. Struct. 17, 015028 (2008).

${ }^{17}$ J. Claracq, J. Sarrazin, and J. Montfor, Rheol. Acta. 43, 38 (2004).

${ }^{18}$ A. J. W. ten Brinke, L. Bailey, H. N. W. Lekkerkerker, and G. C. Maitland, Soft Mater 3, 1145 (2007). 